\title{
CÁC NHÂN TỐ ẢNH HƯỞNG ĐẾN VIỆC VẬN DỤNG KẾ TOÁN QUẢN TRI CHIẾN LƯợC TRONG CÁC DOANH NGHIỆP VIẸT NAM
}

\author{
FACTORS AFFECTING THE IMPLEMENTATION OF STRATEGY \\ MANAGEMENT ACCOUNTING IN VIETNAMESE ENTERPRISES
}

\author{
Trần Hồng Vân, Trần Thị Phương Lan ${ }^{1}$
}

Ngày nhận bài: 14/10/2019 Ngày chấp nhận đăng: 07/11/2019 Ngày đăng: 05/10/2020

\section{Tóm tắt}

Mục đích chính của bài viết là đánh giá ảnh hưởng của các nhân tố đặc điểm doanh nghiệp, cơ cấu tổ chức, chiến lược kinh doanh, định hướng thị trường và kỹ thuật công nghệ thông tin đến việc áp dụng kế toán quản trị chiến lược (Strategic Management Accounting - SMA) tại các doanh nghiệp (DN) Việt Nam. Dữ liệu khảo sát được thu thập từ $311 \mathrm{DN}$, phân bổ ở nhiều địa phương và hoạt động trong các lĩnh vực kinh doanh khác nhau. Kết quả nghiên cứu cho thấy các nhân tố được khảo sát đều ảnh hưởng đến mức độ áp dụng SMA, nhưng các kỹ thuật SMA cụ thể bị ảnh hưởng là khác nhau. Cơ cấu tổ chức, chiến lược kinh doanh và định hướng thị trường ảnh hưởng đến việc áp dụng các kỹ thuật SMA định hướng ra thị trường. Trong khi đó, các nhân tố đặc điểm $\mathrm{DN}$, cơ cấu tổ chức, định hướng thị trường, kỹ thuật công nghệ thông tin ảnh hưởng đến việc áp dụng các kỹ thuật SMA hướng tới chi phí và đánh giá hiệu quả hoạt động. Mặt khác, kết quả nghiên cứu cũng cho thấy, dù số lượng DN áp dụng đầy đủ nội dung của các kỹ thuật SMA chưa cao, nhưng các DN Việt Nam đã quan tâm và có sử dụng kế toán như công cụ để cung cấp thông tin định hướng chiến lược.

Tù khóa: Doanh nghiệp Việt Nam, kế toán quản trị chiến lược, nhân tố ảnh hưởng.

\begin{abstract}
The main purpose of this paper is to evaluate the impact of corporate characteristics, organizational structure, business strategy, market orientation and IT techniques on the implementation of Strategic Management Accounting (SMA) in Vietnamese enterprises. Data were collected from 311 enteprises located in different regions and operating in different businness areas. The results of this study prove that all the factors being examined affect the extent on which SMA is applied, but particular techniques of SMA are affected differently. Organizattional structure, business stratetry and market orientation affect the implementation of market - oriented SMA techniques. Meanwhile, corporate characteristics, organizational structure, market orientation, IT techniques affect SMA techniques that focus on cost and operating efficiency assessment. On the other hand, the results of this study also indicate that the number of enterprises which fully apply the SMA techniques is not very high, but Vietnamese enterprises have had considerations and have used accounting as a tool for providing strategy - orientated information.
\end{abstract}

Keywords: Vietnamese enterprises, strategic Managerial Accounting, factors.

${ }^{1}$ Trường Đại học Tài chính - Marketing 


\section{Giới thiệu}

Áp lực cạnh tranh toàn cầu ngày càng gia tăng, cũng làm gia tăng những thách thức đối với các nhà quản lý, kế toán quản trị vì vậy cũng phải thay đổi để đáp ứng những nhu cầu không ngừng phát sinh và biến đổi của quản trị.

Kế toán quản trị truyền thống thường tập trung đáp ứng những mối quan tâm trong nội bộ $\mathrm{DN}$ và hướng đến cung cấp thông tin tài chính. Trong khi đó, những kỹ thuật kế toán quản trị hiện đại kết hợp cả thông tin tài chính và phi tài chính, đặc biệt tập trung một cách rõ ràng và chi tiết vào chiến lược. Vào những năm cuối thập kỷ 80 của thế kỷ trước, tuy còn khá mới mẻ, kế toán quản trị chiến lược (SMA) đã khẳng định tầm quan trọng.

Tại Việt Nam, mặc dù các công cụ của SMA đã được đưa vào giới thiệu/giảng dạy trong các chương trình chứng chỉ nghề nghiệp kế toán quốc tế hoặc chương trình đào tạo ngành kế toán, nhưng vẫn còn rất ít các công trình nghiên cứu định lượng về mức độ áp dụng SMA, hoặc về các nhân tố ảnh hưởng đến việc áp dụng SMA tại các DN.

Mục đích chính của bài viết này là đánh giá việc áp dụng SMA và xác định mức độ tác động của các nhân tố ngẫu nhiên (đặc điểm $\mathrm{DN}$, cơ cấu tổ chức, chiến lược kinh doanh, định hướng thị trường, kỹ thuật công nghệ thông tin) đến việc áp dụng SMA tại các DN.

\section{Tổng quan nghiên cứu và cơ sở lý thuyết liên quan}

\subsection{Khái niệm $S M A$}

Năm 1981, Simmonds lần đầu tiên đưa ra định nghĩa về SMA trong bài viết "Strategic management accounting" đăng trên Tạp chí Management Accounting số 59 (Roslender \& Hart, 2003; Cinquini \& Tenucci, 2010; Langfield-Smith, 2008). Qua gần 40 năm phát triển, cho đến nay, trong các tài liệu kế toán, các học giả vẫn chưa hoàn toàn thống nhất với nhau về định nghĩa của SMA.

Theo Simmonds, SMA là "việc cung cấp và phân tích dữ liệu kế toán quản trị về doanh nghiệp và các đối thủ cạnh tranh, nhằm sử dụng trong việc phát triển và giám sát chiến lược kinh doanh của doanh nghiệp" (trích dẫn trong Langfield - Smith 2008, trang 205). Ông cho rằng thông tin về đối thủ cạnh tranh (những thông tin liên quan đến chi phí, giá, thị phần,...) có ý nghĩa quan trọng trong quá trình xây dựng và giám sát chiến lược kinh doanh. Sau Simmonds, nhiều học giả tiếp tục ghi nhận vai trò của việc phân tích thông tin về đối thủ cạnh tranh, nhất là trong quá trình tìm kiếm và xây dựng lợi thế cạnh tranh cho doanh nghiệp (Cinquini \& Tenucci, 2010).

Năm 1988, Bromwich (trích dẫn trong Lord 1996, trang 349) lặp lại quan điểm của Simmonds và nhấn mạnh rằng doanh nghiệp nên tập trung vào các vấn đề bên ngoài, vì “doanh nghiệp tạo ra lợi nhuận trên thị trường và các đối thủ cạnh tranh cũng tạo ra thách thức đối với doanh nghiệp trên thị trường này". Bromwich cũng phát triển thêm luận điểm của Simmonds, ông cho rằng không chỉ cần so sánh doanh nghiệp với các đối thủ cạnh tranh mà còn cần đánh giá "lợi ích từ sản phẩm của doanh nghiệp trên góc độ khách hàng và trên góc độ doanh nghiệp".

Trong phạm vi bài viết này, vận dụng quan điểm của Simmonds, Bromwich, nhóm tác giả cho rằng SMA là việc thu thập, xử lý, phân tích dữ liệu kế toán quản trị về $\mathrm{DN}$, đối thủ cạnh tranh, và khách hàng, nhằm sử dụng cho việc phát triển và giám sát chiến lược kinh doanh của DN.

\subsection{Vận dụng SMA trong thục tế}

Mặc dù những định nghĩa và mô tả đối với SMA còn nhiều khác biệt đáng kể, nhưng SMA khi được áp dụng trong thực tế đều sẽ thể hiện ít 
nhất một trong các đặc điểm sau (Guilding et al, 2000): (i) Định hướng ra thị trường/môi trường; (ii) Tập trung vào đối thủ cạnh tranh; và (iii) Định hướng dài hạn.

Các kỹ thuật SMA do Guilding và các cộng sự tổng hợp và đưa ra trong khoảng thời gian 2000-2002 đã lên đến con số 20 (Guilding et al, 2000; Čadež, 2006; Guilding \& McManus, 2002) và được các học giả sử dụng trong nhiều nghiên cứu (một phần hoặc toàn bộ), nhưng đến nay thực tế vẫn chưa có danh sách thống nhất các kỹ thuật SMA được chấp nhận rộng rãi. Nguyên nhân chính dẫn đến điều này là do vẫn chưa có một định nghĩa thống nhất về SMA. Việc xác định các kỹ thuật SMA còn mang tính chủ quan.

Áp dụng các tiêu chí giống Guilding và các cộng sự đã đưa ra khi xác định danh sách các kỹ thuật SMA, trong đó có tham khảo các nghiên cứu khác để tăng/giảm một số kỹ thuật nhằm tránh trùng lắp cũng như tập trung vào các kỹ thuật phù hợp và có khả năng đã được áp dụng trong thực tế các DN Việt Nam, các kỹ thuật SMA được chúng tôi khảo sát trong bài viết này bao gồm: (i) Chi phí thuộc tính (Attribute Costing); (ii) Chi phí mục tiêu (Target Costing); (iii) Chi phí chất lượng (Quality Costing); (iv) Chuẩn đối sánh (Benchmarking); (v) Thẻ điểm cân bằng (Balance Scorecard); (vi) Định giá chiến lược (Strategic Pricing); (vii) Đánh giá chi phí đối thủ cạnh tranh (Competitor Cost Assessment); (viii) Giám sát vị thế cạnh tranh (Competitive Position Monitoring); (ix) Đánh giá đối thủ cạnh tranh dựa trên báo cáo tài chính đã công bố (Competitor Appraisal Based On Published Financial Statements); (x) Phân tích khả năng sinh lợi/sinh lợi suốt đời của khách hàng (Customer Profitability Analysis/Lifetime customer profitability analysis).

\subsection{Giả thuyết nghiên cứu}

Dựa vào lý thuyết bất định và các kết quả nghiên cứu đã được công bố, các nhân tố dự kiến có ảnh hưởng đến mức độ áp dụng SMA tại các DN Việt Nam được khảo sát trong nghiên cứu này bao gồm:

- Đặc điểm DN: Đặc điểm DN trong bài viết này được xem xét ở 02 khía cạnh: quy mô và tuổi của $\mathrm{DN}$. Quy mô $\mathrm{DN}$ được xem là có liên quan trực tiếp đến mức độ phức tạp của hệ thống kế toán. Công ty càng phát triển, các vấn đề phát sinh liên quan đến kiểm soát, thông tin truyền thông càng gia tăng, yêu cầu đối với hệ thống thông tin kế toán càng cao, càng phức tạp. Ngoài ra, quy mô tăng cũng làm giảm chi phí đơn vị của quá trình xử lý thông tin (Cadez \& Guilding, 2008). Nhiều kết quả nghiên cứu đã công bố cũng khẳng định rằng quy mô $\mathrm{DN}$ có tác động tích cực đến mức độ áp dụng các kỹ thuật SMA (Cadez \& Guilding, 2008; Pavlatos, 2015; Cinquini \& Tenucci, 2010). Bên cạnh đó, theo Báo cáo nghiên cứu của Viện Nghiên cứu quản lý kinh tế Trung ương và các tổ chức khác (2016): (i) 85\% DN được điều tra cho biết họ có gặp trở ngại trong kinh doanh, một trong các trở ngại lớn đối với sự phát triển của $\mathrm{DN}$ là mức cạnh tranh quá lớn; (ii) Tỷ lệ DN ra khỏi thị trường ở khu vực “đô thị” (Hà Nội, Hải Phòng và TP.Hồ Chí Minh) nhìn chung là cao hơn và điều này có thể được lý giải bởi áp lực cạnh tranh lớn hơn ở khu vực này. Dựa trên kết quả điều tra $\mathrm{DN}$ này, nhóm tác giả cho rằng các $\mathrm{DN}$ tồn tại càng lâu dài (không bị ra khỏi thị trường) càng có khả năng đã sử dụng nhiều các kỹ thuật quản trị hiện đại, trong đó có các kỹ thuật của SMA. Trên cơ sở đó, về tác động của nhân tố đặc điểm DN đối với việc áp dụng SMA nhóm tác giả cho rằng "Giả thuyết H1: Đặc điểm $D N$ có tác động tỷ lệ thuận với việc áp dụng SMA”.

- Cơ cấu tổ chức: Cơ cấu tổ chức trong bài viết này được xem xét trên hai khía cạnh: mức độ phân quyền quản lý và tính chính thức/minh bạch. Đơn vị càng có mức độ phân quyền cao, các tầng cấp quản lý khác nhau càng cần sự hỗ trợ của SMA trong việc ra các quyết định chiến lược (Pavlatos, 2015). Bên cạnh đó, theo quan 
điểm của nhóm tác giả, nếu công ty quan tâm đến tính chính thức (ban hành dưới dạng văn bản), cụ thể (minh bạch) của quy định, hướng dẫn thì SMA cũng được xem xét công cụ hỗ trợ hữu hiệu. Trên cơ sở này, về tác động của nhân tố cơ cấu tổ chức, nhóm tác giả cho rằng "Giả thuyết H2: Co cấu tổ chức có tác động tỷ lệ thuận với việc áp dụng SMA".

- Chiến lược kinh doanh: Chiến lược kinh doanh được xem là có ảnh hưởng rõ ràng đến việc thiết kế hệ thống kiểm soát (Otley, 2016). Kết quả nghiên cứu trước cho thấy các công ty áp dụng chiến lược Người tiên phong (Prospector Strategy) hoặc Chiến lược chủ động (Deliberate Strategy) có mức độ áp dụng SMA cao hơn các công ty áp dụng các chiến lược ngược lại (Cadez \& Guilding, 2008). Dựa trên kết quả nghiên cứu này, nhóm tác giả cho rằng tính tiên phong, tính chủ động trong chiến lược kinh doanh có tác động tỷ lệ thuận với việc áp dụng SMA tại DN. Giả thuyết H3: Chiến luợc kinh doanh có tác động tỷ lệ thuận với việc áp dụng SMA.

- Định hướng thị trường: Theo Narver và Slater (1990), định hướng thị trường được nhìn nhận ở 3 khía cạnh và 2 dấu hiệu: định hướng khách hàng (customer orientation), định hướng đối thủ cạnh tranh (competitor orientation), phối hợp giữa các bộ phận chức năng (interfunctional coordination), tập trung vào dài hạn (long - term focus) và tối đa hóa lợi nhuận (profit objective). Các kỹ thuật SMA vì vậy rất phù hợp với nhu cầu thông tin hướng ra bên ngoài mà $\mathrm{DN}$ định hướng thị trường cần có. Các công ty có định hướng thị trường cao có xu hướng áp dụng SMA cao hơn (Guilding
\& McManus, 2002; Cadez \& Guilding, 2008). Dựa vào các nghiên cứu trước, nhóm tác giả cho rằng mức độ định hướng thị trường có tác động tỷ lệ thuận với việc áp dụng SMA của DN. Giả thuyết H4: Mức độ định hướng thị truòng có tác động tỷ lệ thuận với việc áp dụng SMA.

- Kỹ thuật công nghệ thông tin: Kỹ thuật công nghệ thông tin được nghiên cứu trong bài viết này được xem xét trên 2 khía cạnh: kỹ thuật công nghệ và công nghệ thông tin. Kỹ thuật công nghệ phát triển sẽ gây ra các khó khăn trong phân bổ chi phí, đánh giá hiệu quả, thẩm định đầu tư. Người quản lý $\mathrm{DN}$ cũng như các kế toán viên phải phát triển các kỹ thuật kế toán mới để xử lý các vấn đề phát sinh này (Kalkhouran et al, 2015). Việc áp dụng kỹ thuật công nghệ mới trong sản xuất cũng làm thay đổi nhu cầu thông tin cần có khi ra các quyết định của nhà quản trị (Isa \& Foong, 2005). Đối với công nghệ thông tin, một số các nghiên cứu đã công bố cũng cho thấy việc có/áp dụng phần mềm, phần cứng, nhân viên công nghệ thông tin giúp DN có lợi thế kinh doanh tốt hơn. Chất lượng của hệ thống thông tin có mối quan hệ tích cực với mức độ áp dụng SMA tại các DN (Rosli et al, 2014; Pavlatos, 2015). Dựa trên các nghiên cứu đã công bố, nhóm tác giả cho rằng kỹ thuật công nghệ thông tin có tác động tỷ lệ thuận với việc áp dụng SMA của DN. Giả thuyết H5: Kỹ thuật công nghệ thông tin có tác động tỷ lệ thuận với việc áp dụng SMA.

\subsection{Mô hình nghiên cứu}

Để xem xét ảnh hưởng của các nhân tố đến mức độ vận dụng SMA, nhóm tác giả sử dụng mô hình như sau (Mô hình 1):

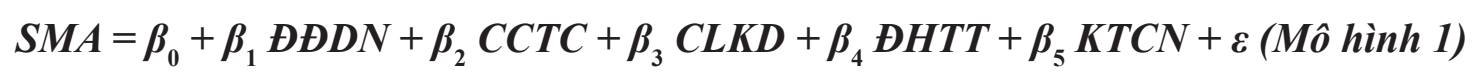

Trong đó: SMA: Mức độ vận dụng SMA; ĐĐDN: Đặc điểm DN; CCTC: Cơ cấu tổ chức; CLKD: Chiến lược kinh doanh; ĐHTT: Định hướng thị trường; KTCN: Kỹ thuật công nghệ thông tin. 


\section{Phương pháp nghiên cứu}

\subsection{Mẫu khảo sát}

Dữ liệu khảo sát được thu thập dưới hai hình thức: phỏng vấn cá nhân (phỏng vấn) và điều tra qua mạng (online). Nhóm tác giả xây dựng một bảng câu hỏi soạn sẵn. Trường hợp phỏng vấn cá nhân, người phỏng vấn sẽ nêu câu hỏi từ bảng câu hỏi chọn sẵn và ghi lại câu trả lời vào phiếu khảo sát. Do phạm vi $\mathrm{DN}$ được khảo sát là trong lãnh thổ Việt Nam nên ngoài các thành viên trong nhóm, nhóm tác giả còn nhờ sự hỗ trợ của một số giảng viên hiện đang tham gia giảng cập nhật kiến thức kế toán hỗ trợ trong việc phỏng vấn trực tiếp. Trường hợp điều tra qua mạng, bảng câu hỏi soạn sẵn được đưa vào google forms. Danh sách email để gửi link google forms khảo sát được lấy từ hai nguồn: (i) email chính thức đã công bố của các công ty niêm yết và (ii) email của cựu sinh viên các khóa/hệ đào tạo của Khoa. DN khảo sát được lựa chọn theo kỹ thuật chọn mẫu thuận tiện. Mỗi một DN chỉ khảo sát một phiếu. Đối tượng trả lời khảo sát là những người làm công tác quản lý (từ trưởng/phó các bộ phận trở lên) hoặc nhân viên kế toán.

\subsection{Thang đo các khái niệm trong mô hình nghiên cứu}

- Mức độ vận dụng SMA: Tên gọi của các kỹ thuật SMA thường ít được biết đến trong thực tế, mặc dù DN đã có vận dụng SMA ở một mức độ nhất định nào đó (Guilding et al, 2000). Ngoài ra, tại Việt Nam, theo nhóm tác giả, khả năng áp dụng đầy đủ nội dung của từng kỹ thuật SMA là không cao, nhưng việc cung cấp thông tin kế toán quản trị hướng ra bên ngoài, hướng ra thị trường, là có, dù ở dạng này hoặc ở dạng khác. Trên cơ sở đó, các câu hỏi khảo sát áp dụng cho thang đo mức độ áp dụng SMA có thay đổi so với Guilding et al (2000), Cinquini và Tenucci (2010), cụ thể: (i) Không liệt kê tên kỹ thuật SMA trong nội dung câu hỏi, thay vào đó, mô tả các bước thực hiện của kỹ thuật; (ii) Thang đo likert 5 mức độ thay đổi từ 1 (hoàn toàn không) đến 5 (mức độ rất lớn) sang 1 (không áp dụng), 2 (áp dụng dưới 50\% nội dung được mô tả, 3 (áp dụng khoảng 50\% nội dung được mô tả), 4 (áp dụng trên $50 \%$ nội dung được mô tả), 5 (áp dụng 100\% nội dung được mô tả).

- Đặc điểm DN: Đặc điểm DN được đo lường thông qua (i) vốn điều lệ, (ii) quy mô hoạt động và (iii) thời gian hoạt động. Biến quan sát ký hiệu là ĐĐDN, bao gồm:

ĐĐDN1: Vốn điều lệ của DN, được chia thành 5 mức độ là 1 (dưới 2 tỷ), 2 (dưới 5 tỷ), 3 (dưới 10 tỷ), 4 (dưới 50 tỷ), 5 (trên 50 tỷ)

ĐĐDN2: Quy mô hoạt động. Do các DN được khảo sát có nhiều hình thức sở hữu khác nhau (DN tư nhân - DNTN, trách nhiệm hữu hạn - TNHH, cổ phần - CP, liên doanh giữa Việt Nam và quốc gia khác - Khác), hoạt động trong nhiều ngành nghề khác nhau, để đo lường quy mô hoạt động, nhóm tác giả sử dụng thang Likert 5 mức độ như sau: 5 (đã hoặc đang nằm trong Bảng xếp hạng VNR500), 4 (công ty đại chúng niêm yết trên HNX, HOSE), 3 (công ty đại chúng, niêm yết UPCOM, OTC), 2 (công ty do cục thuế quản lý), 1 (công ty do chi cục thuế quản lý). Nếu DN thỏa mãn nhiều mức độ thì chọn sử dụng mức độ cao nhất.

ĐĐDN3: Thời gian hoạt động được tính theo công thức "2019 - năm thành lập + 1". Thời gian hoạt động được chia thành 5 mức độ: 1 (dưới 6 năm), 2 (dưới 11 năm), 3 (dưới 16 năm), 4 (dưới 21 năm), 5 (từ 21 năm trở lên).

- Co cấu tổ chức: Dựa trên nội dung khảo sát của Gordon và Narayanan (1984), nhóm tác giả điều chỉnh và trình bày lại các câu hỏi theo thang Likert 5 mức độ, từ 1 (rất thấp) đến 5 (rất cao). Biến quan sát ký hiệu là CCTC, bao gồm:

CCTC1: Mức độ phân quyền thực tế dành cho các nhà quản lý các cấp (trừ cấp rất cao) trong từng mảng công việc phụ trách tương ứng; 
> CCTC2: Mức độ chi tiết, cụ thể trong quy định, hướng dẫn thực hiện và đánh giá hiệu quả đối với mỗi nhiệm vụ, công việc được giao;

CCTC3: Mức độ rõ ràng trong việc phân chia trách nhiệm, quyền hạn giữa các bộ phận, cá nhân;

CCTC4: Mức độ chính thức (bằng văn bản, thông báo công khai,...) của các quy định, hướng dẫn về trách nhiệm, quyền hạn, cách thức thực hiện công việc, đánh giá hiệu quả của nhân viên.

- Chiến lược kinh doanh: Lấy đặc điểm của chiến lược Người tiên phong trong Cinquini và Tenucci (2010) làm cơ sở, nhóm tác giả xây dựng các câu hỏi theo thang Likert 5 mức độ, từ 1 (rất thấp) đến 5 (rất cao). Biến quan sát ký hiệu là CLKD, bao gồm:

CLKD1: Mức độ thay đổi của danh mục các sản phẩm/dịch vụ mà công ty cung cấp ra thị trường qua thời gian;

CLKD2: Vai trò tiên phong của công ty trong việc phát triển các sản phẩm/dịch vụ mới hoặc thị trường mới trong các công ty cùng ngành/cùng lĩnh vực hoạt động;

CLKD3: Khả năng phản ứng của công ty đối với các tín hiệu đầu tiên về nhu cầu hoặc cơ hội của thị trường.

- Định hướng thị trường: Dựa theo nội dung khảo sát của Guilding và McManus (2002), nhóm tác giả điều chỉnh và trình bày lại các câu hỏi theo thang Likert 5 mức độ, từ 1 (rất thấp) đến 5 (rất cao). Biến quan sát ký hiệu là ĐHTT, bao gồm:

ĐHTT1: Mức độ hiểu biết của công ty về khách hàng của mình;

ĐHTT2: Mức độ chặt chẽ trong việc phối hợp các hoạt động trong công ty để tạo ra giá trị vượt trội cho khách hàng;
> ĐHTT3: Tầm quan trọng của việc phục vụ nhu cầu và mong muốn của thị trường nhằm đạt được sự phát triển và lợi nhuận dài hạn cho công ty trong nhận thức của các nhà quản trị tại công ty;

ĐHTT4: Mức độ rõ nét của việc công ty theo định hướng thị trường.

- Kỹ thuật công nghệ thông tin: Dựa trên nội dung khảo sát của Ojra (2014), nhóm tác giả điều chỉnh và trình bày lại các câu hỏi theo thang Likert 5 mức độ, từ 1 (rất thấp) đến 5 (rất cao). Biến quan sát ký hiệu là $\mathrm{CN}$, bao gồm:

CN1: Mức độ sử dụng công nghệ trong hệ thống hoạt động của công ty;

CN2: Mức độ sử dụng công nghệ trong kỹ thuật sản xuất sản phẩm/dịch vụ của công ty;

CN3: Mức độ áp dụng máy tính vào hệ thống thông tin kế toán của công ty;

CN4: Mức độ phù hợp của các phần mềm hỗ trợ cho công tác kế toán và các hoạt động khác trong công ty.

\section{Kết quả và thảo luận}

\subsection{Thống kê DN được khảo sát}

Quá trình khảo sát được thực hiện từ ngày 01/06/2019 đến ngày 30/09/2019. Tổng số phiếu khảo sát thu về (online và phỏng vấn) là 368 (online: 107, phỏng vấn: 261). Trong đó, loại 57 phiếu trả lời phỏng vấn do không đủ các thông tin cần thiết, giữ lại 311 phiếu. Các DN được khảo sát trải rộng trong cả nước, nhưng chiếm số đông là có trụ sở chính tại TP. Hồ Chí Minh (214 DN, chiếm 68,8\%). Đối tượng trả lời phỏng vấn chủ yếu là các kế toán viên (238 phiếu, chiếm 76,5\%).

Dựa trên mã số thuế của các $\mathrm{DN}$ có được từ các phiếu khảo sát, nhóm tác giả xác định lĩnh vực hoạt động kinh doanh chính dựa trên ngành nghề đã đăng ký trên giấy phép kinh doanh và 
tiến hành phân loại theo Quyết định số 27/2018/ QĐ-Ttg (Bảng 1). Ngành có số lượng phiếu khảo sát thu về cao nhất là bán buôn và bán lẻ; sửa chữa ô tô, mô tô, xe máy và xe có động cơ khác (107/311, chiếm 34,4\%), xếp thứ 2 là công nghiệp chế biến, chế tạo (82/311, chiếm $26,4 \%)$.

Bảng 1. Lĩnh vực hoạt động kinh doanh chính của các DN được khảo sát

\begin{tabular}{|c|l|c|}
\hline STT & \multicolumn{1}{|c|}{ Ngành } & Tổng \\
\hline 1 & Công nghiệp chế biến, chế tạo & 82 \\
\hline 2 & Xây dựng & 29 \\
\hline 3 & Bán buôn và bán lẻ; sửa chữa ô tô, mô tô, xe máy và xe có động cơ khác & 107 \\
\hline 4 & Vận tải kho bãi & 16 \\
\hline 5 & Hoạt động kinh doanh bất động sản & 12 \\
\hline 6 & Hoạt động chuyên môn, khoa học và công nghệ & 19 \\
\hline 7 & Hoạt động hành chính và dịch vụ hỗ trợ & 16 \\
\hline 8 & Lĩnh vực hoạt động khác & 30 \\
\hline Tổng & & 311 \\
\hline
\end{tabular}

Nguồn: Kết quả tính toán tù̀ SPSS 20

Thống kê mức độ vận dụng SMA tại các DN (Bảng 2) cho thấy mức độ bình quân của ĐG_BCTC là thấp nhất $(2,87)$, cao nhất là $\mathrm{PT}$ SL $(3,33)$, nhưng đều chỉ giao động trên dưới mức "áp dụng khoảng 50\% nội dung được mô tả”. Tuy nhiên, đã có khá nhiều DN thực hiện $100 \%$ nội dung được mô tả, cao nhất là $\mathrm{CP}$ CHLUONG (43 DN, chiếm 16.4\%), thấp nhất là DG_BCTC (18 DN, chiếm 13.8\%).

Bảng 2. Mức độ vận dụng SMA tại các DN

\begin{tabular}{|c|c|c|c|c|c|c|c|c|c|}
\hline \multirow{2}{*}{$\begin{array}{l}\text { Số } \\
\text { TT }\end{array}$} & \multirow[t]{2}{*}{ KỸ THUẬT SMA } & \multirow[t]{2}{*}{ KÝ HIỆU } & \multirow{2}{*}{$\begin{array}{l}\text { Mức } \\
\text { bình } \\
\text { quân }\end{array}$} & \multicolumn{6}{|c|}{$\begin{array}{l}\text { Số DN theo từng mức độ } \\
\text { áp dụng }\end{array}$} \\
\hline & & & & 1 & 2 & 3 & 4 & 5 & Tổng \\
\hline 1 & Chi phí thuộc tính & CP_TTINH & 2.95 & 33 & 78 & 96 & 80 & 24 & 311 \\
\hline 2 & Chi phí mục tiêu & CP_MTIEU & 3.22 & 19 & 52 & 106 & 110 & 24 & 311 \\
\hline 3 & Chi phí chất lượng & CP_CHLUONG & 3.29 & 23 & 54 & 87 & 104 & 43 & 311 \\
\hline 4 & Chuẩn đối sánh & BENCH & 2.98 & 24 & 76 & 113 & 78 & 20 & 311 \\
\hline 5 & Thẻ điểm cân bằng & $\mathrm{BCS}$ & 3.15 & 16 & 66 & 110 & 92 & 27 & 311 \\
\hline 6 & Định giá chiến lược & DG_CL & 3.29 & 17 & 55 & 99 & 101 & 39 & 311 \\
\hline 7 & Đánh giá chi phí đối thủ cạnh tranh & DG_DT & 3.03 & 18 & 73 & 126 & 70 & 24 & 311 \\
\hline 8 & Giám sát vị thế cạnh tranh & GS_CT & 3.11 & 17 & 66 & 122 & 79 & 27 & 311 \\
\hline 9 & $\begin{array}{l}\text { Đánh giá đối thủ cạnh tranh dựa } \\
\text { trên báo cáo tài chính đã công bố }\end{array}$ & DG_BCTC & 2.87 & 38 & 72 & 112 & 71 & 18 & 311 \\
\hline 10 & $\begin{array}{l}\text { Phân tích khả năng sinh lợi/sinh } \\
\text { lợi suốt đời của khách hàng }\end{array}$ & PT_SL & 3.33 & 15 & 50 & 103 & 102 & 41 & 15 \\
\hline
\end{tabular}




\subsection{Kết quả nghiên cứu}

\subsection{1. Đo lường độ tin cậy cho thang đo}

Thực hiện kiểm định độ tin cậy thang đo Cronbach's Alpha trong SPSS 20 cho các nhóm biến quan sát, kết quả cho thấy: (i) tất cả các biến đều có hệ số tương quan tổng phù hợp $(\geq 0.3)$; và (ii) hệ số Cronbach's Alpha của các nhóm biến quan sát đều đạt yêu cầu về độ tin cậy $(\geq 0.6)$. Như vậy, các biến quan sát đều có thể đưa vào phân tích nhân tố khám phá EFA.

\subsubsection{Phân tích nhân tố khám phá EFA}

Tiến hành phân tích nhân tố khám phá cho các biến độc lập, kết quả cho thấy (Bảng 3):

\section{Bảng 3. Kết quả phân tích nhân tố khám phá EFA}

\begin{tabular}{|c|c|c|c|c|}
\hline \multirow[b]{2}{*}{ Phân tích EFA } & \multicolumn{2}{|r|}{ Biến độc lập } & \multicolumn{2}{|r|}{ Biến phụ thuộc } \\
\hline & $\begin{array}{l}\text { Kết } \\
\text { quả }\end{array}$ & Kết luận & $\begin{array}{l}\text { Kết } \\
\text { quả }\end{array}$ & Kết luận \\
\hline $\begin{array}{l}\text { Hệ số KMO } \\
(0.5 \leq \mathrm{KMO} \leq 1)\end{array}$ & 0.889 & $\begin{array}{l}\text { Phân tích nhân tố được chấp } \\
\text { nhận }\end{array}$ & 0.872 & $\begin{array}{l}\text { Phân tích nhân tố được chấp } \\
\text { nhận }\end{array}$ \\
\hline $\begin{array}{l}\text { Sig Barlett's } \\
\text { Test }(<0.05)\end{array}$ & .000 & Phân tích nhân tố là phù hợp & .000 & Phân tích nhân tố là phù hợp \\
\hline $\begin{array}{l}\text { Eigenvalues } \\
(\geq 1)\end{array}$ & 1.073 & $\begin{array}{l}\text { Trích được } 5 \text { nhân tố mang } \\
\text { ý nghĩa tóm tắt thông tin tốt } \\
\text { nhất }\end{array}$ & 1.245 & $\begin{array}{l}\text { Trích được } 2 \text { nhân tố mang } \\
\text { ý nghĩa tóm tắt thông tin tốt } \\
\text { nhất }\end{array}$ \\
\hline $\begin{array}{l}\text { Tổng phương } \\
\text { sai trích } \\
(\geq 50 \%)\end{array}$ & 64.926 & $\begin{array}{l}\text { Mô hình EFA là phù hợp. } 5 \\
\text { nhân tố được trích cô đọng } \\
\text { được } 64.926 \text { biến thiên các } \\
\text { biến quan sát. }\end{array}$ & 54.094 & $\begin{array}{l}\text { Mô hình EFA là phù hợp. } 2 \\
\text { nhân tố được trích cô đọng } \\
\text { được } 54.094 \text { biến thiên các } \\
\text { biến quan sát }\end{array}$ \\
\hline $\begin{array}{l}\text { Kết quả ma } \\
\text { trận xoay }\end{array}$ & $\begin{array}{c}\text { Xem } \\
\text { Bảng } \\
4 \mathrm{a}\end{array}$ & $\begin{array}{l}18 \text { biến quan sát được gom } \\
\text { thành } 5 \text { nhân tố, tất cả các } \\
\text { biến quan sát đều có hệ số tải } \\
\text { nhân tố Factor Loading lớn } \\
\text { hơn } 0.5\end{array}$ & $\begin{array}{c}\text { Xem } \\
\text { Bảng } \\
4 b\end{array}$ & $\begin{array}{l}10 \text { biến quan sát được gom } \\
\text { thành } 2 \text { nhân tố, tất cả các } \\
\text { biến quan sát đều có hệ số tải } \\
\text { nhân tố Factor Loading lớn } \\
\text { hơn } 0.5\end{array}$ \\
\hline
\end{tabular}

Kết quả phân tích EFA cho thấy các biến quan sát thuộc các thang đo ĐHTT, CN, CCTC, ĐĐDN, CLKD không thay đổi. Riêng các biến quan sát của thang đo Kỹ thuật vận dụng SMA bị tách ra thành 2 nhấn tố: (1) gồm DG_DT, DG_BCTC, GS_CT, DG_CL, PT_SL, chủ yếu là các kỹ thuật hướng đến kế toán đối thủ cạnh tranh, khách hàng, ra quyết định giá; và (2) gồm CP_MTIEU, CP TTINH, BCS, BENCH, CP CHLUONG là các kỹ thuật hướng đến xác định chi phí - giá thành và phân tích hiệu quả hoạt động. Theo kết quả phân tích nhân tố khám phá EFA, các thang đo được định nghĩa lại (Bảng 5): 
Bảng 5. Bảng tổng hợp thang đo mới

\begin{tabular}{|c|l|l|l|}
\hline STT & Thang đo & \multicolumn{1}{|c|}{ Các biến quan sát } & \multicolumn{1}{c|}{ Tên thang đo } \\
\hline 1 & ĐHTT & ĐHTT1, ĐHTT2, ĐHTT4, ĐHTT3 (4 biến) & Định hướng thị trường \\
\hline 2 & CN & CN3, CN4, CN2, CN1 (4 biến) & Kỹ thuật công nghệ thông tin \\
\hline 3 & CCTC & CCTC4, CCTC3, CCTC2, CCTC1 (4 biến) & Cơ cấu tổ chức \\
\hline 4 & DDDN & DDDN2, DDDN1, DDDN3 (3 biến) & Đặc điểm DN \\
\hline 5 & CLKD & CLKD1, CLKD2, CLKD3 (3 biến) & Chiến lược kinh doanh \\
\hline 6 & SMA_1 & $\begin{array}{l}\text { DG_DT, DG_BCTC, GS_CT, DG_CL, } \\
\text { PT_SL (5 biến) }\end{array}$ & $\begin{array}{l}\text { Mức độ vận dụng SMA - Định } \\
\text { hướng thị trường }\end{array}$ \\
\hline 7 & SMA_2 & $\begin{array}{l}\text { CP_MTIEU, CP_TTINH, BCS, BENCH, } \\
\text { CP_CHLUONG (5 biến) }\end{array}$ & $\begin{array}{l}\text { Mức độ vận dụng SMA - Định } \\
\text { hướng chi phí và đánh giá hoạt } \\
\text { động }\end{array}$ \\
\hline
\end{tabular}

Bảng 4a. Ma trận xoay các biến quan sát độc lập

Rotated Component Matrix ${ }^{a}$

\begin{tabular}{|l|c|c|c|c|c|}
\hline & \multicolumn{5}{|c|}{ Component } \\
\cline { 2 - 6 } & 1 & 2 & 3 & 4 & 5 \\
\hline DHTT1 & .737 & & & & \\
DHTT2 & .713 & & & & \\
DHTT3 & .678 & & & & \\
CN3 & .663 & & & & \\
CN4 & & .801 & & & \\
CN1 & & .743 & & & \\
CN2 & & .655 & & & \\
CCTC4 & & & .712 & & \\
CCTC3 & & & .703 & & \\
CCTC2 & & & .686 & & \\
CCTC1 & & & .637 & & \\
DDDN2 & & & & .835 & \\
DDDN1 & & & & .803 & \\
DDDN3 & & & & .634 & \\
CLKD1 & & & & & \\
CLKD2 & & & & & \\
CLKD3 & & & & & \\
\hline Extraction & & & & \\
\end{tabular}

Extraction Method: Principal Component Analysis.

Rotation Method: Varimax with Kaiser Normalization. a. Rotation converged in 7 iterations.

\subsubsection{Phân tích hồi quy tuyến tính}

Do mức độ vận dụng SMA được tách thành 2 thang đo mới nên:

+ Các giả thuyết từ $\mathrm{H} 1$ đến $\mathrm{H} 5$ được xem xét riêng cho từng nhóm kỹ thuật SMA, cụ thể:
Bảng 4b. Ma trận xoay các biến quan sát phụ thuộc

Rotated Component Matrix ${ }^{a}$

\begin{tabular}{|l|r|r|}
\hline & \multicolumn{2}{|c|}{ Component } \\
\cline { 2 - 3 } & 1 & \multicolumn{2}{|c|}{2} \\
DG_DT & .832 & \\
DG_BCTC & .798 & \\
GS_CT & .749 & \\
DG_CL & .578 & \\
PT_SL & .532 & \\
CP_MTIEU & & .740 \\
CP_TTINH & & .683 \\
BCS & & .679 \\
BENCH & & .663 \\
CP_CHLUONG & & .609 \\
\hline
\end{tabular}

Extraction Method: Principal Component Analysis.

Rotation Method: Varimax with Kaiser Normalization. a. Rotation converged in 3 iterations.

Nguồn: Tổng hợp tù̀ SPSS 20

$\checkmark$ Giả thuyết H1a: Đặc điểm DN có tác động tỷ lệ thuận với việc áp dụng SMA - Định hướng thị trường; Giả thuyết H1b: Đặc điểm DN có tác động tỷ lệ thuận với việc áp dụng SMA Định hướng chi phí và đánh giá hoạt động. 
$\checkmark$ Giả thuyết H2a: Cơ cấu tổ chức có tác động tỷ lệ thuận với việc áp dụng SMA - Định hướng thị trường; Giả thuyết $\mathbf{H 2 b}$ : Cơ cấu tổ chức có tác động tỷ lệ thuận với việc áp dụng SMA Định hướng chi phí và đánh giá hoạt động.

Giả thuyết H3a: Chiến lược kinh doanh có tác động tỷ lệ thuận với việc áp dụng SMA Định hướng thị trường; Giả thuyết H3b: Chiến lược kinh doanh có tác động tỷ lệ thuận với việc áp dụng SMA - Định hướng chi phí và đánh giá hoạt động.

Giả thuyết H4a: Mức độ định hướng thị trường có tác động tỷ lệ thuận với việc áp dụng
SMA - Định hướng thị trường; Giả thuyết H4b: Mức độ định hướng thị trường có tác động tỷ lệ thuận với việc áp dụng SMA - Định hướng chi phí và đánh giá hoạt động.

$\checkmark$ Giả thuyết H5a: Kỹ thuật công nghệ thông tin có tác động tỷ lệ thuận với việc áp dụng SMA - Định hướng thị trường; Giả thuyết H5b: Kỹ thuật công nghệ thông tin có tác động tỷ lệ thuận với việc áp dụng SMA - Định hướng chi phí và đánh giá hoạt động.

+ Mô hình nghiên cứu dự kiến ban đầu (Mô hình 1) được thay bằng 02 mô hình hồi quy:

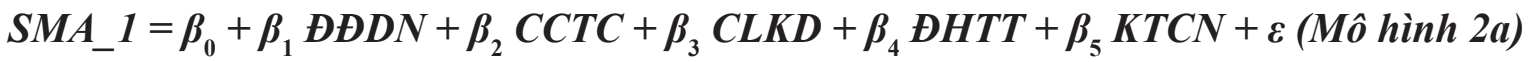

$$
\begin{aligned}
& S M A_{-} 2=\beta_{0}+\beta_{1} Đ Ð D N+\beta_{2} C C T C+\beta_{3} C L K D+\beta_{4} \boxplus H T T+\beta_{5} K T C N+\varepsilon(M \hat{o} \operatorname{hìh} 2 b)
\end{aligned}
$$

Trong đó: SMA_1: Mức độ vận dụng các kỹ thuật SMA_1; SMA_2: Mức độ vận dụng các kỹ thuật SMA_2; ĐĐDN: Đặc điểm DN ; CCTC: Cơ cấu tổ chức; CLKD: Chiến lược kinh doanh; ĐHTT: Định hướng thị trường; KTCN: Kỹ thuật công nghệ thông tin.

\subsubsection{Kiểm định Mô hình hồi quy 2a}

Thực hiện phân tích hồi quy Mô hình 2a, giá trị phân tích hồi quy hầu hết đều đạt yêu cầu, riêng Sig kiểm định $\mathrm{t}$ hệ số hồi quy của biến $\mathrm{CN}$ $=0.214>0.05$ và biến ĐĐDN $=0.088>0.05$ nên phải loại ra khỏi mô hình. Loại hai biến $\mathrm{CN}$ và ĐĐDN ra khỏi mô hình, kết quả phân tích hồi quy như sau (Bảng 6): (i) Giá trị $\mathrm{R}^{2}$ hiệu chỉnh $=0.295$ : Biến độc lập đưa vào chạy hồi quy ảnh hưởng $29.5 \%$ sự thay đổi của biến phụ thuộc, còn lại 70.5\% là do các biến ngoài mô hình và sai số ngẫu nhiên; (ii) Hệ số DurbinWatson $=1.812$ : Không có hiện tượng tự tương quan chuỗi bậc nhất xảy ra; (iii) Sig kiểm định $\mathrm{F}=0.00<0.05$ : Mô hình hồi quy tuyến tính phù hợp với tập dữ liệu và có thể sử dụng được; (iv) Sig kiểm định $t$ hệ số hồi quy của các biến độc lập đều nhỏ hơn 0.05: Các biến độc lập đều có ý nghĩa giải thích cho biến phụ thuộc, không có biến nào bị loại khỏi mô hình; (v) Tất cả giá trị sig mối tương quan hạng giữa ABSRES với các biến độc lập đều lớn hơn 0.05: Phương sai phần dư là đồng nhất, giả định phương sai không đổi không bị vi phạm; (vi) Các hệ số hồi quy đều lớn hơn 0 : Các biến độc lập đưa vào phân tích hồi quy đều tác động cùng chiều tới biến phụ thuộc. Dựa vào độ lớn của hệ số hồi quy chuẩn hóa Beta, thứ tự tác động từ mạnh nhất tới yếu nhất của các biến độc lập đến biến phụ thuộc SMA_1 là: CLKD (0.249) > ĐHTT (0.214) > CCTC (0.199). 
Bảng 6. Kết quả phân tích hồi quy Mô hình 2a sau khi loại bỏ biến CN và biến DDDN

Model Summary ${ }^{b}$

\begin{tabular}{|l|r|r|r|r|r|}
\hline Model & \multicolumn{1}{|c|}{$\mathrm{R}$} & R Square & $\begin{array}{c}\text { Adjusted R } \\
\text { Square }\end{array}$ & $\begin{array}{c}\text { Std. Error of } \\
\text { the Estimate }\end{array}$ & Durbin-Watson \\
\hline 1 & $.543^{\mathrm{a}}$ & .295 & .288 & .65135 & 1.812 \\
\hline
\end{tabular}

a. Predictors: (Constant), CLKD, CCTC, ĐHTT

b. Dependent Variable: SMA1

ANOVA ${ }^{a}$

\begin{tabular}{|rl|r|r|r|r|r|}
\hline Model & \multicolumn{1}{c|}{$\begin{array}{c}\text { Sum of } \\
\text { Squares }\end{array}$} & \multicolumn{1}{c|}{ df } & Mean Square & F & Sig. \\
\hline 1 & Regression & 54.582 & 3 & 18.194 & 42.884 & $.000^{\mathrm{b}}$ \\
\hline & Residual & 130.248 & 307 & .424 & & \\
\hline Total & 184.829 & 310 & & & \\
\hline
\end{tabular}

a. Dependent Variable: SMA1

b. Predictors: (Constant), CLKD, CCTC, ĐHTT

Coefficients $^{\mathrm{a}}$

\begin{tabular}{|c|c|c|c|c|c|c|c|c|c|c|}
\hline \multirow{2}{*}{ Moc } & & \multicolumn{2}{|c|}{$\begin{array}{c}\text { Unstandardized } \\
\text { Coefficients } \\
\end{array}$} & \multirow{2}{*}{$\begin{array}{c}\text { Standardized } \\
\text { Coefficients } \\
\text { Beta }\end{array}$} & \multirow[t]{2}{*}{$\mathrm{t}$} & \multirow[t]{2}{*}{ Sig. } & \multicolumn{2}{|c|}{$\begin{array}{c}95.0 \% \text { Confidence } \\
\text { Interval for B } \\
\end{array}$} & \multicolumn{2}{|c|}{$\begin{array}{c}\text { Collinearity } \\
\text { Statistics } \\
\end{array}$} \\
\hline & & B & $\begin{array}{l}\text { Std. } \\
\text { Error }\end{array}$ & & & & $\begin{array}{l}\text { Lower } \\
\text { Bound }\end{array}$ & $\begin{array}{l}\text { Upper } \\
\text { Bound }\end{array}$ & Tolerance & VIF \\
\hline \multirow{4}{*}{1} & (Constant) & .560 & .231 & .214 & 2.418 & .016 & .104 & 1.015 & & \\
\hline & DHTT & .260 & .074 & .199 & 3.514 & .001 & .114 & .405 & .620 & 1.613 \\
\hline & ССТC & .223 & .066 & .249 & 3.400 & .001 & .094 & .352 & .667 & 1.499 \\
\hline & CLKD & .277 & .065 & & 4.285 & .000 & .150 & .404 & .678 & 1.475 \\
\hline
\end{tabular}

Correlations

\begin{tabular}{|c|c|c|c|c|c|c|}
\hline & & & ABSRES & ĐHTT & СCTC & CLKD \\
\hline \multirow{12}{*}{ Spearman's rho } & \multirow{3}{*}{ ABSRES } & Correlation Coefficient & 1.000 & .005 & .071 & -.105 \\
\hline & & Sig. (2-tailed) & & .934 & .209 & .064 \\
\hline & & $\mathrm{N}$ & 311 & 311 & 311 & 311 \\
\hline & \multirow{3}{*}{ ĐHTT } & Correlation Coefficient & .005 & 1.000 & $.536^{* *}$ & $.477^{* *}$ \\
\hline & & Sig. (2-tailed) & .934 & . & .000 & .000 \\
\hline & & $\mathrm{N}$ & 311 & 311 & 311 & 311 \\
\hline & \multirow{3}{*}{ CCTC } & Correlation Coefficient & .071 & $.536^{* *}$ & 1.000 & $.437^{* *}$ \\
\hline & & Sig. (2-tailed) & .209 & .000 & . & .000 \\
\hline & & $\mathrm{N}$ & 311 & 311 & 311 & 311 \\
\hline & \multirow{3}{*}{ CLKD } & Correlation Coefficient & -.105 & $.477^{* *}$ & $.437^{* *}$ & 1.000 \\
\hline & & Sig. (2-tailed) & .064 & .000 & .000 & \\
\hline & & $\mathrm{N}$ & 311 & 311 & 311 & 311 \\
\hline
\end{tabular}

**. Correlation is significant at the 0.01 level (2-tailed). 
Với 5 giả thuyết từ $\mathrm{H} 1 \mathrm{a}$ đến $\mathrm{H} 5 \mathrm{a}$, có 3 giả thuyết được chấp nhận $\mathrm{H} 2 \mathrm{a}, \mathrm{H} 3 \mathrm{a}, \mathrm{H} 4 \mathrm{a}$, tương ứng: Cơ cấu tổ chức, Chiến lược kinh doanh và Định hướng thị trường có ảnh hưởng đến mức độ vận dụng các kỹ thuật SMA- định hướng thị trường. Giả thuyết H1a và H5a bị bác bỏ, nhân tố Đặc điểm doanh nghiệp và Kỹ thuật công nghệ thông tin không có ý nghĩa trong mô hình hồi quy. Phương trình hồi quy chuẩn hóa: SMA_1 $=0.199 *$ CCTC $+0.249 *$ CLKD $+0.214 *$ ĐHTT.

\subsubsection{Kiểm định Mô hình hồi quy $2 b$}

Thực hiện phân tích hồi quy Mô hình $2 b$, giá trị phân tích hồi quy cho kết quả đạt yêu cầu, tuy nhiên, giá trị sig mối tương quan hạng giữa $\mathrm{ABSRES}$ với biến $\mathrm{CLKD}=0.047<0.05$ : giả định phương sai không đổi bị vi phạm nên phải loại ra khỏi mô hình. Loại biến CLKD ra khỏi mô hình, kết quả phân tích hồi quy như sau (Bảng 7): (i) Giá trị $\mathrm{R}^{2}$ hiệu chỉnh = 0.423: Biến độc lập đưa vào chạy hồi quy ảnh hưởng $42.3 \%$ sự thay đổi của biến phụ thuộc, còn lại $57.7 \%$ là do các biến ngoài mô hình và sai số ngẫu nhiên; (ii) Hệ số Durbin-Watson = 2.026: Không có hiện tượng tự tương quan chuỗi bậc nhất xảy ra; (iii) Sig kiểm định $\mathrm{F}=0.00<0.05$ : Mô hình hồi quy tuyến tính phù hợp với tập dữ liệu và có thể sử dụng được; (iv) Sig kiểm định thệ số hồi quy của các biến độc lập đều nhỏ $\leq$ 0.05: Các biến độc lập đều có ý nghĩa giải thích cho biến phụ thuộc, không có biến nào bị loại khỏi mô hình; (v) Tất cả giá trị sig mối tương quan hạng giữa $\mathrm{ABSRES}$ với các biến độc lập đều lớn hơn 0.05: Phương sai phần dư là đồng nhất, giả định phương sai không đổi không bị vi phạm; (vi) Các hệ số hồi quy đều lớn hơn 0 : Các biến độc lập đưa vào phân tích hồi quy đều tác động cùng chiều tới biến phụ thuộc. Dựa vào độ lớn của hệ số hồi quy chuẩn hóa Beta, thứ tự tác động từ mạnh nhất tới yếu nhất của các biến độc lập đến biến phụ thuộc SMA_2 là: $\mathrm{CN}(0.267)>\operatorname{DDDN}(0.220)>\operatorname{CCTC}(0.182)>$ DHTT (0.161).

Với 5 giả thuyết từ $\mathrm{H} 1 \mathrm{~b}$ đến $\mathrm{H} 5 \mathrm{~b}$, có 4 giả thuyết được chấp nhận H1b, H2b, H4b, H5b tương ứng: Đặc điểm DN, Cơ cấu tổ chức, Định hướng thị trường, Kỹ thuật công nghệ thông tin có ảnh hưởng đến mức độ vận dụng các kỹ thuật SMA- Định hướng chi phí và đánh giá hoạt động. Giả thuyết H3b phải loại ra khỏi mô hình hồi quy vì giá trị Sig tương quan Spearman giữa phần dư chuẩn hóa với nhân tố Chiến lược kinh doanh nhỏ hơn 0.05 , nếu để lại sẽ làm kết quả của phương trình hồi quy không chính xác, dẫn đến lệch kết quả so với thực tế. Phương trình hồi quy chuẩn hóa: SMA_2 $=\mathbf{0 . 2 2} * \mathrm{DDDN}+$ $0.182 *$ CCTC $+0.161 *$ DHTT $+0.267 *$ CN.

\section{Bảng 7. Kết quả phân tích hồi quy Mô hình 2b sau khi loại bỏ biến CLKD}

\begin{tabular}{|llrrrr|}
\multicolumn{7}{c|}{ Model Summary $^{\mathbf{b}}$} \\
\hline Model & R & R Square & $\begin{array}{c}\text { Adjusted R } \\
\text { Square }\end{array}$ & $\begin{array}{l}\text { Std. Error of } \\
\text { the Estimate }\end{array}$ & Durbin-Watson \\
\hline 1 & $.656^{\mathrm{a}}$ & .431 & .423 & .57480 & 2.026 \\
\hline
\end{tabular}

a. Predictors: (Constant), DDDN, DHTT, CCTC, CN

b. Dependent Variable: SMA2

\section{ANOVA $^{a}$}

\begin{tabular}{|llrrrrr|}
\hline Model & & Sum of Squares & df & Mean Square & F & Sig. \\
\hline 1 & Regression & 76.544 & 4 & 19.136 & 57.919 & $.000^{\mathrm{b}}$ \\
& Residual & 101.101 & 306 & .330 & & \\
& Total & 177.646 & 310 & & & \\
\hline
\end{tabular}

a. Dependent Variable: SMA2

b. Predictors: (Constant), DDDN, DHTT, CCTC, CN 
Coefficients $^{\mathbf{a}}$

\begin{tabular}{|c|c|c|c|c|c|c|c|c|c|c|}
\hline \multirow{2}{*}{\multicolumn{2}{|c|}{ Model }} & \multicolumn{2}{|c|}{$\begin{array}{c}\text { Unstandardized } \\
\text { Coefficients }\end{array}$} & \multirow{2}{*}{$\begin{array}{c}\begin{array}{c}\text { Standardized } \\
\text { Coefficients }\end{array} \\
\text { Beta }\end{array}$} & \multirow{2}{*}{$\mathrm{t}$} & \multirow{2}{*}{ Sig. } & \multicolumn{2}{|c|}{$\begin{array}{c}95.0 \% \text { Confidence } \\
\text { Interval for B }\end{array}$} & \multicolumn{2}{|c|}{$\begin{array}{c}\text { Collinearity } \\
\text { Statistics }\end{array}$} \\
\hline & & B & $\begin{array}{l}\text { Std. } \\
\text { Error }\end{array}$ & & & & $\begin{array}{l}\text { Lower } \\
\text { Bound }\end{array}$ & $\begin{array}{l}\text { Upper } \\
\text { Bound }\end{array}$ & Tolerance & VIF \\
\hline \multirow[t]{5}{*}{1} & (Constant) & .310 & .203 & & 1.527 & .128 & -.089 & .709 & & \\
\hline & DHTT & .192 & .067 & .161 & 2.853 & .005 & .060 & .324 & .582 & 1.719 \\
\hline & $\mathrm{CN}$ & .280 & .062 & .267 & 4.531 & .000 & .159 & .402 & .537 & 1.863 \\
\hline & ССТC & .199 & .062 & .182 & 3.207 & .001 & .077 & .321 & .580 & 1.724 \\
\hline & DDDN & .170 & .039 & .220 & 4.389 & .000 & .094 & .246 & .737 & 1.356 \\
\hline
\end{tabular}

a. Dependent Variable: SMA2

\section{Correlations}

\begin{tabular}{|c|c|c|c|c|c|c|c|}
\hline & & & ABSRES & DHTT & $\mathrm{CN}$ & CCTC & DDDN \\
\hline \multirow{15}{*}{ Spearman's rho } & \multirow{3}{*}{ ABSRES } & Correlation Coefficient & 1.000 & -.063 & -.009 & -.014 & .026 \\
\hline & & Sig. (2-tailed) & & .268 & .879 & .808 & .653 \\
\hline & & $\mathrm{N}$ & 311 & 311 & 311 & 311 & 311 \\
\hline & \multirow{3}{*}{ DHTT } & Correlation Coefficient & -.063 & 1.000 & $.582^{* *}$ & $.536^{* *}$ & $.366^{* *}$ \\
\hline & & Sig. (2-tailed) & .268 & & .000 & .000 & .000 \\
\hline & & $\mathrm{N}$ & 311 & 311 & 311 & 311 & 311 \\
\hline & \multirow{3}{*}{$\mathrm{CN}$} & Correlation Coefficient & -.009 & $.582^{* *}$ & 1.000 & $.584^{* *}$ & $.443^{* *}$ \\
\hline & & Sig. (2-tailed) & .879 & .000 & & .000 & .000 \\
\hline & & $\mathrm{N}$ & 311 & 311 & 311 & 311 & 311 \\
\hline & \multirow{3}{*}{ CCTC } & Correlation Coefficient & -.014 & $.536^{* *}$ & $.584^{* *}$ & 1.000 & $.482^{* *}$ \\
\hline & & Sig. (2-tailed) & .808 & .000 & .000 & & .000 \\
\hline & & $\mathrm{N}$ & 311 & 311 & 311 & 311 & 311 \\
\hline & \multirow{3}{*}{ DDDN } & Correlation Coefficient & .026 & $.366^{* *}$ & $.443^{* *}$ & $.482^{* *}$ & 1.000 \\
\hline & & Sig. (2-tailed) & .653 & .000 & .000 & .000 & \\
\hline & & $\mathrm{N}$ & 311 & 311 & 311 & 311 & 311 \\
\hline
\end{tabular}

**. Correlation is significant at the 0.01 level (2-tailed).

\section{Kết luận}

Mặc dù số lượng doanh nghiệp áp dụng hoàn toàn kỹ thuật SMA còn hạn chế, nhưng đa số các kỹ thuật SMA đều được áp dụng bình quân trên $50 \%$ nội dung như mô tả. Các doanh nghiệp đã hướng tới việc áp dụng kế toán quản trị như là một công cụ để cung cấp thông tin phục vụ cho việc đưa ra các quyết định chiến lược của công ty.

Các nhân tố Đặc điểm doanh nghiệp, Cơ cấu tổ chức, Chiến lược kinh doanh, Định hướng thị trường, Kỹ thuật công nghệ thông tin có ảnh hưởng đến mức độ áp dụng kỹ thuật SMA tại các doanh nghiệp Việt Nam nói chung, tuy nhiên có thay đổi giữa các nhóm kỹ thuật khác nhau.
Cơ cấu tổ chức và Định hướng thị trường có ảnh hưởng tích cực đến mức độ áp dụng của tất cả các kỹ thuật SMA được khảo sát. Trong khi đó, nhân tố Chiến lược kinh doanh chưa chứng minh được là có ảnh hưởng đến mức độ áp của các kỹ thuật SMA nhằm cung cấp thông tin chi phí và phân tích hiệu quả hoạt động, nhưng lại có ảnh hưởng mạnh nhất đối với việc áp dụng các kỹ thuật SMA hướng ra thị trường. Tương tự như vậy, nhân tố Đặc điểm doanh nghiệp và nhân tố Kỹ thuật công nghệ chưa thể hiện có ảnh hưởng đến mức độ áp dụng của các kỹ thuật SMA hướng ra thị trường, nhưng hai nhân tố này có ảnh hưởng rất mạnh đến việc áp dụng các kỹ thuật SMA hướng đến chi phí và phân tích hiệu quả hoạt động. 


\section{Tài liệu tham khảo}

Narver, J. C., and Slater, S. F. (1990). The Effect of a Market Orientation on Business Profitability. Journal of Marketing, 54(4), 20. doi:10.2307/1251757

Isa, C. R., and Foong, S.-Y. (2005). Adoption of advanced manufacturing technology (AMT) and management accounting practices: the case of manufacturing firms in Malaysia. World Review of Science, Technology and Sustainable Development, 2(1), 35. doi:10.1504/wrstsd.2005.006726

Cadez, S. (2006). A Cross-Industry Comparison Of Strategic Management Accounting Practices: An Exploratory Study. Economic and business review for Central and South-Eastern Europe, 8 (3), 279298.

Cadez, S., and Guilding, C. (2008). An exploratory investigation of an integrated contingency model of strategic management accounting. Accounting, Organizations and Society, 33(7-8), 836-863. doi:10.1016/j.aos.2008.01.0031o.

Rosli, M. H., Said, J., and Mohd, F. (2014). Factors that influence the use of Strategic Management Accounting (SMA) in Malaysian Government-Linked companies (GLCs). Malaysian Accounting Review, 13(2), 23-46. Available at: http://arionline.uitm.edu.my/ojs/index.php/MAR/article/view/32.

Pavlatos, O. (2015). An empirical investigation of strategic management accounting in hotels. International Journal of Contemporary Hospitality Management, 27(5), 756-767. doi:10.1108/ijchm-12-2013-0582.

Kalkhouran, A. A. N., Rasid, S. Z. A., Sofian, S., and Nedaei, B. H. N. (2015). A Conceptual Framework for Assessing the Use of Strategic Management Accounting in Small and Medium Enterprises. Global Business and Organizational Excellence, 35(1), 45-54. doi:10.1002/joe.21644.

Cinquini,L., and Tenucci,A.(2010). Strategic managementaccounting and business strategy:Aloose coupling? Journal of Accounting \& Organizational Change, 6(2), 228-259. doi:10.1108/18325911011048772.

Gordon, L. A., and Narayanan, V. K. (1984). Management accounting systems, perceived environmental uncertainty and organization structure: An empirical investigation. Accounting, Organizations and Society, 9(1), 33-47. doi:10.1016/0361-3682(84)90028-x.

Guilding, C., Cravens, K.S., and Tayles, M. (2000). An International Comparison Of Strategic Manangement Accounting Practices. Management Accounting Research, 11,113-135. doi: 10.1006/mare.1999.0120.

Guilding, C., and McManus, L. (2002). The incidence, perceived merit and antecedents of customer accounting: an exploratory note. Accounting, Organizations and Society, 27(1-2), 45-59. doi:10.1016/ s0361-3682(01)00030-7.

Langfield-Smith, K. (2008). Strategic management accounting: how far have we come in 25 years? Accounting, Auditing \& Accountability Journal, 21(2), 204-228. doi:10.1108/09513570810854400

Lord, B. R. (1996). Strategic Management Accounting: The Emperor's New Clothes? Management Accounting Research, 7(3), 347-366. doi:10.1006/mare.1996.0020.

Ojra, J. (2014). Strategic Management Accounting Practices in Palestinian Companies: Application of Contingency Theory Perspective. PhD Thesis, University of East Anglia. Available at: https://core. ac.uk/download/pdf/29107927.pdf.

Otley, D. (2016). The contingency theory of management accounting and control: 1980-2014. Management Accounting Research, 31, 45-62. doi:10.1016/j.mar.2016.02.001.

Roslender, R., and Hart, S. J. (2003). In search of strategic management accounting: theoretical and field study perspectives. Management Accounting Research, 14(3): 255-279. doi:10.1016/s10445005(03)00048-9.

Thủ tướng Chính phủ. (2018). Quyết định số 27/2018/QĐ-Ttg Quyết định Ban hành Hệ thống ngành kinh tế Việt Nam. Available at: http://vbpl.vn/TW/Pages/vbpq-van-ban-goc.aspx?ItemID=130098.

Viện Nghiên cứu quản lý kinh tế Trung ương, Bộ Kế hoạch và Đầu tư, Đại học Unu-Wider, Viện Khoa học Lao động và Xã hội. (2016). Báo cáo đặc điểm môi trường kinh doanh Việt Nam: Kết quả điều tra doanh nghiệp nhỏ và vừa năm 2015. Available at: https://www.wider.unu.edu/sites/default/files/ SME2015-report-Vietnamese.pdf. 